\title{
Gérontologie et gériatrie du fond de l'œil
}

\begin{tabular}{|l|l|}
\hline B. & Streiff $^{\mathrm{a}}$ \\
\hline J. & Babel $^{\mathrm{b}}$ \\
\hline
\end{tabular}

Lausanne ${ }^{\mathrm{b}}$ Genève

Adresse des auteurs: Prof. Dr. E. B. Streiff. Clinique universitaire d'Ophtalmologie, Lausanne (Suisse) et Dr. J. Babel, Clinique universitaire d'Ophtalmologie, Genève (Suisse)

Dans ce rapport qui sera publié in extenso dans les Progrès en Ophtalmologie, vol. XIII, on donne un aperçu des alterations gérontologiques et gériatriques des vaisseaux rétiniens, de la rétine, de la macula, de Гépithélium pigmentaire, de la choroïde, du nerí optique et des fonctions visuelles. Cette etude est illustrée par des figures ophtalnioscopiques, biomicroscopiques et histologiques, et se base également sur des statistiques en partie personnelles.

Parmi les alterations gérontologiques on discute Гatteinte vas-culaire, Гaspect du tissu rétinien, les kystes de Blessig-Ivanow, la raréfication du pigment, le fundus tabulatus. les verrucosités de la lame vitrée on druses, Гexcavation du nerf optique. Les fonctions visuelles (acuité, adaptation, sens chromatique, champ vi-suel, champ du regard) sont étudiées d'après les résultats des recherches actuelles.

Parmi les manifestations gériatriques on décrit les lesions sclé-reuses vasculaires, les troubles consécutifs et leur pathogénie, les différents depots qui s'observent dans la rétine, la dyshorie, la ca-pillarose, la choroïditis guttata, la rétinite circinée, les trous iamel-laires périphériques, les trous vrais, le retinoschisis, la chorio-rétinopathie maculaire sèche el humide, la dégénérescence disci-forme, la pseudotumeur senile, la dégénérescence kystoïde de la macula, le trou lamellaire. On signale les facteurs héréditaires dans les dégénérescences maculaires. On étudie encore la dégénérescence pigmentaire senile périphérique, les stries moniliformes de la choriorétine, la sclérose choroïdienne périphérique diffuse el centrale areolaire, Гatteinte du nerf optique par artériosclérose (forme atrophique avec on sans excavation glaucomateuse), la ma-ladie de Horton. Enfin on donne un aperçu des différents traite-ments préconisés pour les alterations gériatriques. 